\title{
On the fractal dimension of small-scale magnetic structures in the Sun
}

\author{
K. Janßen ${ }^{1}$, A. Vögler ${ }^{2}$, and F. Kneer ${ }^{1}$ \\ 1 Universitäts-Sternwarte, Geismarlandstraße 11, 37083 Göttingen, Germany \\ ${ }^{2}$ Max-Planck-Institut für Aeronomie, Max-Planck-Straße 2, 37191 Katlenburg-Lindau, Germany
}

Received 11 June 2003 / Accepted 29 July 2003

\begin{abstract}
We compare, by means of fractal analyses, the shapes of observed small-scale magnetic structures on the Sun with those of magnetic features resulting from numerical simulations of magnetoconvection. The observations were obtained with the "Göttingen" Fabry-Perot spectrometer at the Vacuum Tower Telescope at the Observatorio del Teide on Tenerife. Magnetograms with 0!'4-0'.5 spatial resolution were obtained from two-dimensional Stokes $V$ polarimetry in the Fe I $6302.5 \AA$ line and by image reconstruction with speckle methods. The simulations of magnetoconvection was performed with the MURAM code. It solves the time-dependent MHD equations for a compressible, partly ionized plasma including radiative transfer in LTE. To determine the fractal dimensions the perimeter-area relation is used. We discuss the influence of seeing and noise in the fractal dimension $D$ of the observed magnetograms. A dependence of $D$ on the distance from disk center could not be found. The observations give $D=1.21 \pm 0.05$ for a pixel size corresponding to 0 ' 105 , while for the numerical simulations $D=1.38 \pm 0.07$ for a pixel size of $20.83 \mathrm{~km}$. If we use a yardstick adapted to the spatial resolution the observations give the dimension $D=$ $1.41 \pm 0.05$ in close agreement with the simulations. This agreement is remarkable since the pixel sizes and spatial resolutions of the simulations and of the observations differ by a factor of 15 . The finding supports the view of self-similarity of solar magnetic structures over a large range of scales. In addition, it demonstrates the realism of the simulations and suggests that all important physical processes are included. We discuss our results in comparison with other investigations.
\end{abstract}

Key words. Sun: magnetic fields - Sun: photosphere - Sun: granulation

\section{Introduction}

In a recent series of publications, Stenflo \& Holzreuter (2002, $2003 \mathrm{a}, \mathrm{b})$ have drawn attention to the astounding distribution of magnetic field structures on the Sun. On very different scales, as seen in Kitt Peak magnetograms, in MDI magnetograms from SOHO, and in magnetograms with today's highest spatial resolution, from the Swedish Vacuum Solar Telescope (SVST) on La Palma, the magnetic patches present themselves with very similar shapes, i.e. they exhibit frayed out borders. When zooming and going to smaller details, one obtains the same picture of rugged structures.

Moreover, as Stenflo \& Holzreuter (2002, 2003a) point out, the probability distribution functions (PDFs) for magnetic field strengths possess closely agreeing shapes irrespectively of the spatial resolution of the magnetograms. The authors ascribe this scale invariance to the nature of turbulent magnetoconvection. They predict that the fractal-like self-similarity will

Send offprint requests to: K. Janßen, e-mail: kjanssen@uni-sw.gwdg.de continue down to the dissipation scales (Stenflo \& Holzreuter 2003b). The same picture will arise whatever the spatial resolution will be.

Fractals on the Sun have already been investigated several times in studies of the granulation and of magnetic fields. An adequate means to express the complexity of structures and their underlying physical dynamics like granules consists in the fractal dimension $D$ measured from the area-perimeter relation of structures (Mandelbrot 1977)

$P^{1 / D} \propto A^{1 / 2}$ or $\frac{1}{D} \log P=\frac{1}{2} \log A+$ const

with $P=$ perimeter and $A=$ area of the structures. Selfsimilarity, or geometric similarity, is expressed by a linear relationship between $\log P$ and $\log A$ over some range of scales.

The fractal dimension of granular perimeters has been measured by Roudier \& Muller (1987). From Pic du Midi data, they find $D=1.25$ for granular diameters of $d \leq 1$ ". 37 and $D \approx 2$ for larger granules. Karpinsky (1990) has evaluated granulation images obtained with the Soviet Stratospheric Solar Observatory and found $D=1.18$ for diameters $d \leq 11^{\prime \prime} 88$ and $D \geq 2$ for $d \geq 1$ "' 88 . Greimel et al. (1990) presented results from the German Spektro-Stratoskop with $D=1.3$ for small 
scales and $D \approx 2$ for larger granules. Likewise, Hirzberger et al. (1997) deduce from SVST data $D=1.3$ for $d \leq 1$ ". 4 and $D \approx 2$ for $d \geq 1$.'7. The latter authors note that the dimension $D \approx 1.3 \approx 4 / 3$ is expected from isotropic and homogeneous turbulence in an isobaric atmosphere. The large value $D \approx 2$ for large granules expresses the high complexity of these structures. Seemingly, there is a break in the geometric similarity at scales of 1 .' 4 to 1 .' 8 .

It is found that the dimension $D$ of granules depends on the manner how the perimeters in the granular intensity pattern are defined. Bovelet \& Wiehr (2001) applied a "multiple level tracking" algorithm to speckle reconstructed images obtained with the Vacuum Tower Telescope (VTT) on Tenerife. They obtain $D=1.09$ for all scales, i.e. $D$ is close to one and shows no increase towards large structures. Similarly, when drawing the perimeters of granular cells in the intergranular lanes between intensity inflection points, Hirzberger et al. (1997) find $D=1.16$, which is again low and demonstrates that intergranular lanes are smooth.

A prescription differing from Eq. (1) to measure a fractal dimension $D^{\prime}$ of magnetic structures in high-resolution magnetograms was used by Tarbell et al. (1990), Schrijver et al. (1992), and Balke et al. (1993). For a given threshold of the magnetic field $\left|B_{\mathrm{th}}\right|$ they determine the number of contiguous pixels, i.e. the area of the magnetic structure, and the minimum size $L$ of a square box containing the structure. The dimension is then defined by

$L^{D^{\prime}} \propto A$ or $D^{\prime} \log L=\log A+$ const $^{\prime}$.

This relation measures the fractal dimension of the area $A$, while Eq. (1) gives the dimension of the perimeter $P$. For simple structures like circles, rectangles, etc. Eq. (1) yields $D=1.0$ and Eq. (2) results $D^{\prime}=2.0$.

Tarbell et al. (1990) and Balke et al. (1993) give values of the area dimension of structures with $L \leq 3^{\prime \prime}$ of $D^{\prime}=1.54$. The latter authors note that the area-length relation cannot be described by a single dimension for the whole range of scales of magnetic clusters.

As in Stenflo \& Holzreuter (2002, 2003a), MDI magnetograms were already used by Meunier (1999) for an extended fractal analysis. In her contribution to the study of the formation of active regions, she applied both definitions of the fractal dimension, Eqs. (1) and (2) above. We will repeatedly comment on Meunier's (1999) work and results below.

In the present contribution we study the geometric structure of magnetic fields also by means of a fractal analysis. We compare observed, high spatial resolution magnetograms with magnetograms from numerical simulations. Sections 2 and 3 deal with the observations and the data analysis, respectively, and Sect. 4 describes the simulations. We discuss and compare the results of the fractal analysis of the observations and simulations in Sect. 5 and give conclusions in Sect. 6. It should be emphasized that we measure the fractal dimension $D$ of the perimeter $P$ according to Eq. (1) which is distinct from the dimension determined also for magnetic field structures by Tarbell et al. (1990) and Balke et al. (1993).

\section{Observations}

The observations stem from a campaign carried out with the Vacuum Tower Telscope (VTT) at the Observatorio del Teide on Tenerife in August 2000. Their details are described in Janßen (2003).

We concentrated on weak activity regions and enhanced network at various positions on the solar disk with $\cos \vartheta$ ranging from 0.2 to $0.99(\vartheta=$ angle between line-of-sight and normal to the solar surface). The targets were selected by means of video imaging in the $G$ band.

The "Göttingen" Fabry-Perot interferometric spectrometer (FPI) was used with the setup described in Koschinsky et al. (2001). It is based on the work of Bendlin et al. (1992), Bendlin (1993), and Bendlin \& Volkmer (1995). The spectrometer includes a Stokes $V$ polarimeter which separates the two circularly polarized beams into two adjacent light paths. Thus the two images containing $\frac{1}{2}\left(I_{\lambda}+V_{\lambda}\right)$ and $\frac{1}{2}\left(I_{\lambda}-V_{\lambda}\right)$, respectively, are formed on separate areas of a CCD detector. The fields of view $(F O V)$ were approximately $23^{\prime \prime} \times 15^{\prime \prime}$ and the image scale was $0 . ' 105$ per pixel.

With the narrow-band channel of the FPI the Fe I $6302.5 \AA$ line (Landé factor $g=2.5$ ) was scanned. Seven short exposures $(40 \mathrm{~ms})$ were taken at 21 wavelength positions giving 147 frames per scan. The scanning across the whole spectral range of $617 \mathrm{~m} \AA$ took $24 \mathrm{~s}$.

From the same $F O V$, broadband frames through a $100 \AA$ wide filter centered at $6300 \AA$ were obtained strictly simultaneously with the narrow-band images, with the identical exposure time. For the data analysis, dark frames and flat field scans were taken as well, the latter with both either fast moving pointing or defocussed telescope from quiet Sun disk center.

\section{Data analysis}

The pre-analysis contained subtraction of average dark frames, shifts to maximum correlation of the frames from one wavelength scan, and flat fielding.

\subsection{Image reconstruction}

The broadband images from each scan were reconstructed with the "Göttingen" speckle code (cf. de Boer et al. 1992; de Boer 1996). It employs the spectral ratio method by von der Lühe (1984) and the speckle masking by Weigelt (1977) and Weigelt \& Wirnitzer (1983).

This reconstructed image represents an estimate of the broadband scene in the FOV and is used to deconvolve the narrow-band images containing the spectral and the polarimetric information (see Keller \& von der Lühe 1992; Krieg et al. 1999; Koschinsky et al. 2001). We obtain thus at each pixel in the $F O V$ the profiles $\frac{1}{2}\left(I_{\lambda}+V_{\lambda}\right)$ and $\frac{1}{2}\left(I_{\lambda}-V_{\lambda}\right)$. The estimated, noise limited spatial resolution in the final magnetograms (see below) is the same as in Koschinsky et al. (2001), approximately $0 . ' 4$. 


\subsection{Polarimetry and magnetic fields}

The flat fielding and further treatment of the narrow-band data is very entangled and laborious. The separate contribution by Janßen (2003) deals in depth with the problems. The main tasks consisted in several steps:

1. proper alignment of the geometric FOVs in the two $\frac{1}{2}\left(I_{\lambda}+\right.$ $\left.V_{\lambda}\right)$ and $\frac{1}{2}\left(I_{\lambda}-V_{\lambda}\right)$ channels for a correct addition and subtraction to retrieve $I_{\lambda}$ and $V_{\lambda}$;

2. proper alignment in wavelength of the two channels and their normalization;

3. correction of the wavelength shift across the $F O V$ (the FPIs are mounted in the collimated beam);

4. proper division by the wavelength dependent gain table keeping the wavelength information in the data.
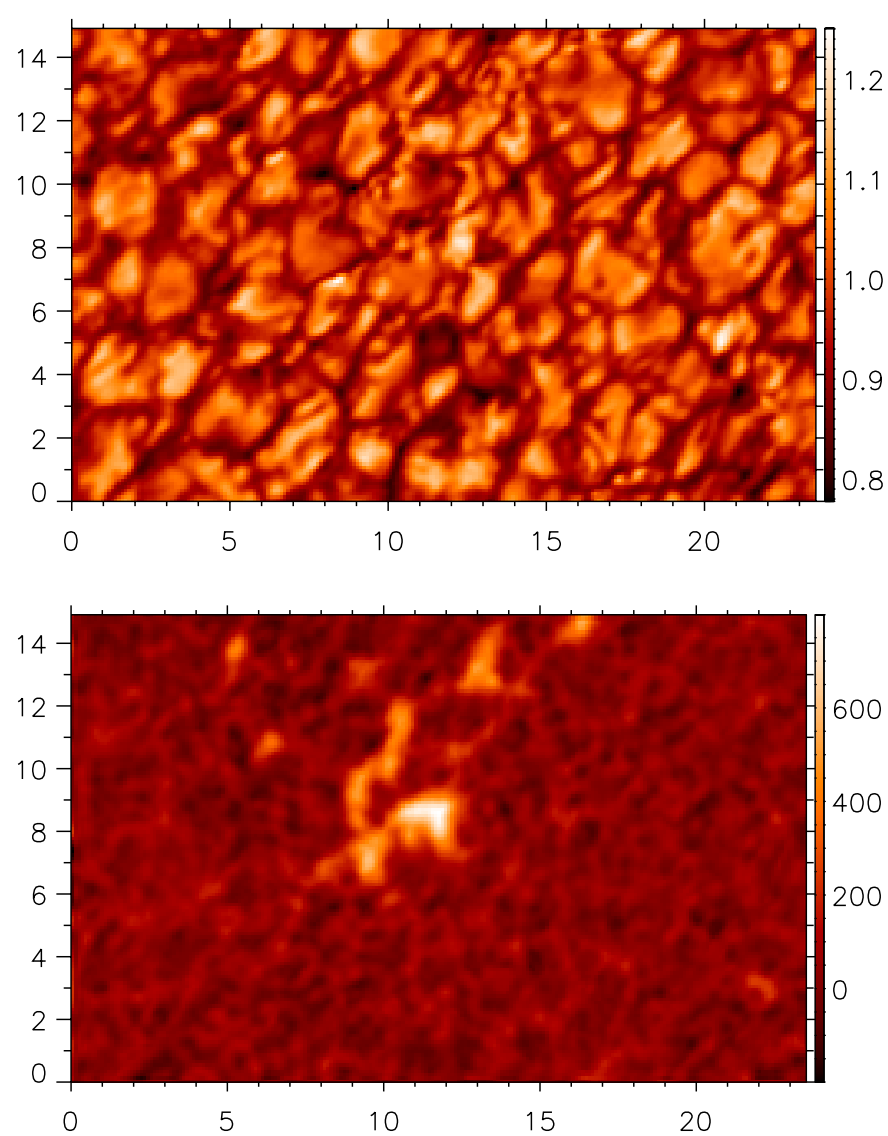

Fig. 1. Reconstructed broadband image (upper panel) and magnetogram (lower panel), coded according to the bar in Gauss, obtained at $\cos \vartheta=0.82$. The tickmarks are at $1^{\prime \prime}$ distance.

The polarimetric signals are then used to derive the magnetic flux along the line-of-sight within each pixel, i.e. an average flux density. We used two methods for the determination of the average field strength $B_{\text {eff }}$ :

1. the center of gravity (COG) method by Semel (1967) and Rees \& Semel (1979) which measures the (average) field component along the line-of-sight from the displacement of the $\frac{1}{2}\left(I_{\lambda}+V_{\lambda}\right)$ and $\frac{1}{2}\left(I_{\lambda}-V_{\lambda}\right)$ profiles;
2. the method based on the Stokes $V$ amplitudes assuming the validity of the weak field approximation $(W F A)$ and the calibration in Koschinsky et al. (2001, Fig. 2 there).

Figure 2 in Koschinsky et al. (2001) shows that for intrinsic field strengths $\left|B_{\text {true }}\right| \leq 1000$ Gauss the separation of the $C O G$ s is proportional to the $V$ amplitude. Yet the $W F A$ does not apply to the strong fields in the network boundaries and plage regions. Besides, recent studies by Domínguez Cerdeña et al. (2003a, 2003b) have revealed kGauss fields in intra-network regions. We thus discard for the present study the magnetic flux determinations from the $V$ amplitudes and concentrate on the $C O G$ measurements. In the latter, the magnetic field signal stems from a formation height of the wavelength average of the $\frac{1}{2}\left(I_{\lambda}+V_{\lambda}\right)$ and $\frac{1}{2}\left(I_{\lambda}-V_{\lambda}\right)$ profiles, which we estimate to be located close to or lower than $100 \mathrm{~km}$ (above $\tau_{5000}=1$ ).

As in Koschinsky et al. (2001) a polarimetric sensitivity of $V_{\lambda} / I_{\mathrm{c}} \approx 0.01$ was achieved. The detection limit of flux densities from the $C O G$ method is $\left|B_{\text {eff }}\right| \approx 50$ Gauss.

Figure 1 gives an example of a granular scene and of the magnetic field structures with its field strengths. The maximum $\left|B_{\text {eff }}\right|$ here amounts to 800 Gauss.

\section{Numerical simulations}

The numerical data used in Sect. 5 were taken from MHD simulations of photospheric magnetoconvection done with the MURAM code.

The MURAM code is the result of a joint venture of the MHD simulation groups at MPAe/Katlenburg-Lindau and at the University of Chicago. It solves the time-dependent MHD equations for a compressible and partially ionized plasma with radiative transfer. The radiative transfer equation is treated under the assumption of local thermodynamic equilibrium; the frequency dependence is taken into account through opacity binning. The bottom boundary condition allows for free in- and outflow of matter, maintaining a constant total mass in the box as well as a fixed energy flux through the system, while the top boundary is closed. The magnetic field is assumed to be vertical at the top and bottom boundaries, the footpoints of fieldlines are allowed to move freely. The horizontal directions are taken to be periodic. A detailed description of the code and a discussion of the magnetoconvection simulations carried out with it can be found in Vögler et al. (2003), Vögler \& Schüssler (2003), Schüssler (2003) and Vögler (2003).

The dimensions of the computational domain of the simulation used here are $1400 \mathrm{~km}$ in the vertical direction and $6000 \mathrm{~km}$ in both horizontal directions, with a resolution of $100 \times 288 \times 288$ grid points. The simulation starts from a planeparallel model of the solar atmosphere extending from $800 \mathrm{~km}$ below to $600 \mathrm{~km}$ above the level of continuum optical depth unity at $5000 \AA$. After convection has fully developed, a homogeneous vertical initial field of 50 Gauss is introduced. Within a few minutes of simulated time (approximately one turnover time of the convection) most of the magnetic flux is swept into the intergranular downflow lanes. Larger magnetic structures are formed at granule vertices where several downflow lanes merge. Figure 2 shows an exemplary simulation snapshot of the 

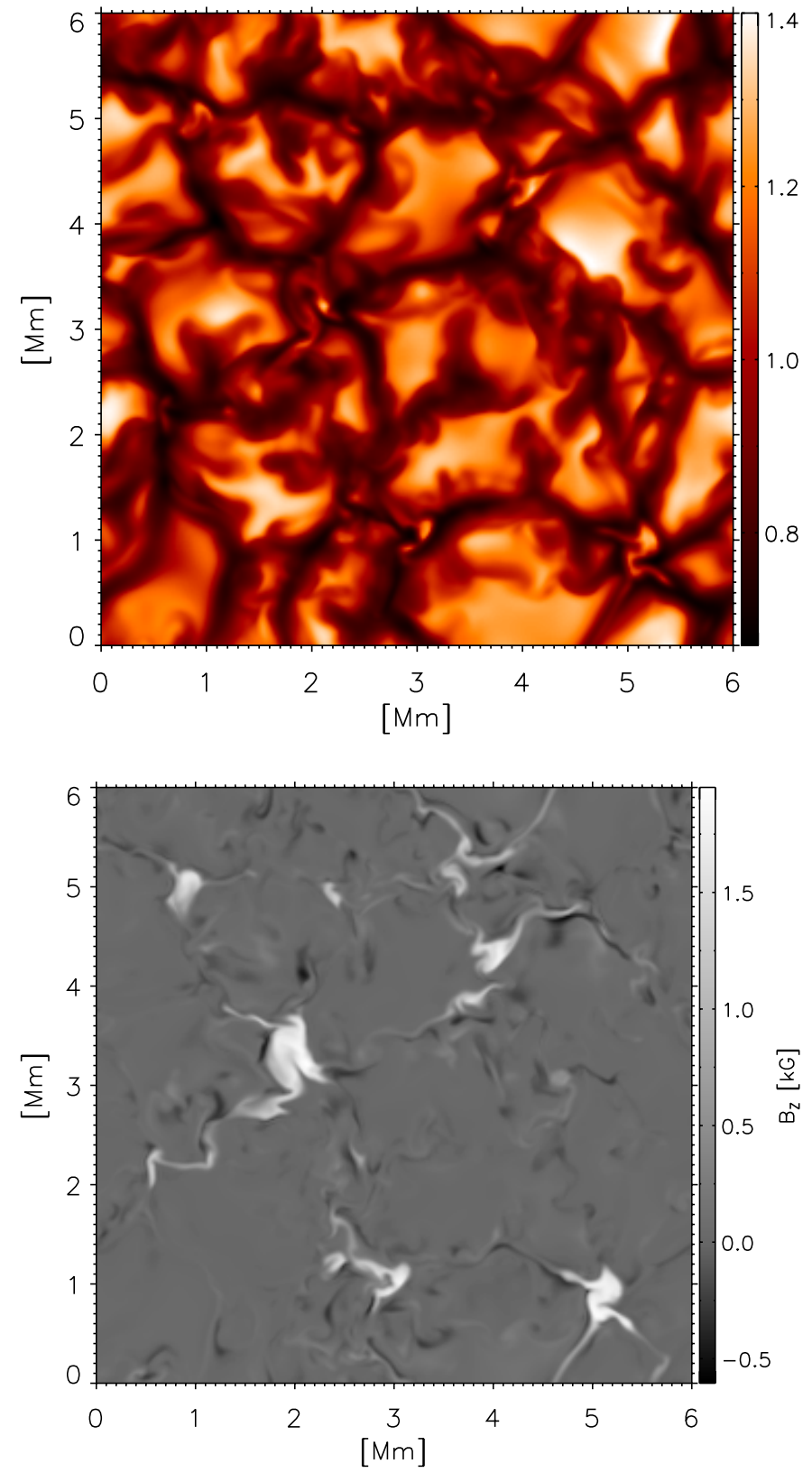

Fig. 2. Snapshot from a numerical simulation of magnetoconvection with an average vertical field of 50 Gauss. Upper panel: frequencyintegrated intensity; lower panel: vertical magnetic field component $B_{\mathrm{z}}$ at a height corresponding to $\tau_{5000}=1$. The pixel size is $20.83 \mathrm{~km}$, the simulated area is $6000 \times 6000 \mathrm{~km}^{2}$.

granular pattern and the magnetic field distribution. The magnetic field maps used to determine the fractal dimension were taken from a geometrical height level which approximately corresponds to $\tau_{5000}=1$.

\section{Fractal dimensions of observations and simulations}

\subsection{Measuring perimeter and area}

The finding of structural borders in magnetograms is less involved than for the granular pattern. A lower limit of $\left|B_{\text {eff }}\right|$ is given and it is asked whether the modulus of the fieldstrength in the pixel under evaluation exceeds this threshold. If not stated otherwise, we chose $\left|B_{\text {eff }}\right|=120$ Gauss, well above the noise limit. The perimeters are measured by adding the lengths of the pixel sides along the outer contours of the magnetic patches. In the determinations of the fractal dimension $D$ we excluded magnetic structures having less than six contiguous pixels fulfilling the above criterion. Choosing a minimum of eight pixels gave very similar results but reduced the statistical sample.

Before presenting the results of dimension measurements we note an important invariance which is often overlooked. Geometric (self-)similarity of structures of different scales requires that the dimension $D$ be independent of the yardstick length $G$ (Mandelbrot 1977, p. 110). We shall expand on this point below. Our length $G$ will be the side length of one pixel, sometimes multiples of it. Thus, $G$ corresponds to $0.1(\approx 72.5 \mathrm{~km})$ for the observations and to $20.83 \mathrm{~km}$ for the numerical simulations.

\subsection{Observations}

\subsubsection{Center-to-limb variation (CLV)}

While $\operatorname{linear} \log (P)$ vs. $\log (A)$ relations will be presented below, we start here with the CLV of $D$ which is depicted in Fig. 3. Apart from the observation at $\cos \vartheta=0.2$, where only 12 magnetic structures were found, all other data give $D$ in the range of 1.16 to 1.22 . There is only a weak dependence on $\cos \vartheta$, if at all. Such a behaviour is expected when only foreshortening plays a role. In this case $P$ and $A$ are diminished by different factors, but the two factors are independent of the size of the structures. Thus, only the constant term (const) in Eq. (1) changes with the distance from disk center. However, when observing near the solar limb one probes higher layers than near disk center. Obviously, the height difference has little influence on the complexity of the magnetic structures. The magnetic fields have not (yet) merged into simpler shaped structures when probing layers $50-100 \mathrm{~km}$ higher than at deep photospheric layers.

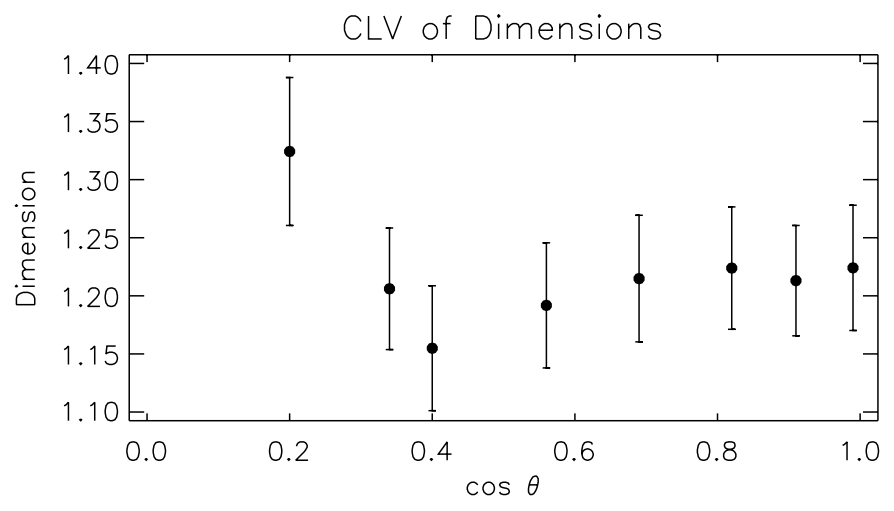

Fig. 3. Center-to-limb variation of the fractal dimension $D$. A threshold of $\left|B_{\text {eff }}\right|=120$ Gauss for including pixels in the structures was used.

Given the very small dependence on the disk position and to increase the statistical significance, we combined all data. Figure 4 shows the $P-A$ relation from the measurements of 


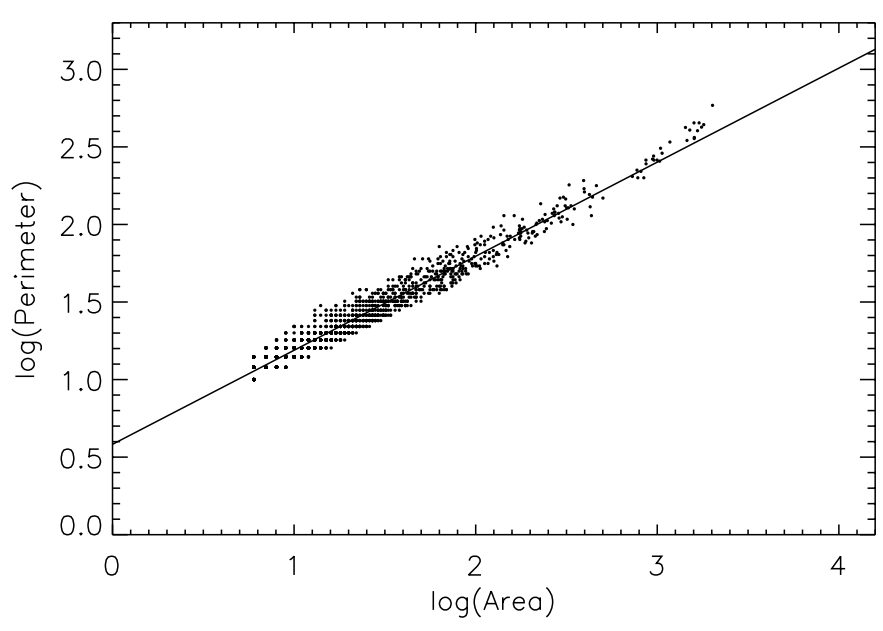

Fig. 4. Perimeter-area relation from 2015 magnetic structures giving the fractal dimension $D=1.21 \pm 0.05$. The perimeter is given in units of pixels, the area in pixel ${ }^{2}$. Here the pixel size is $72 \mathrm{~km}$.

2015 structures. The fitted straight line in Fig. 4 gives $D=$ $1.21 \pm 0.05$. There is a small tendency of increased dimension for structural areas with $\log \left(A /\right.$ pixel $\left.^{2}\right) \geq 2.5$. We will discuss this behaviour below.

Meunier (1999), in her fractal dimension analysis of MDI data, used both full disk (FD) magnetograms with a spatial resolutions of $4^{\prime \prime}$ and high resolution (HR) magnetograms with a resolution of 1 ". 25 . The FD data give dimensions which depend on the area itself, i.e. a non-perfect geometric similarity, and on the magnetic threshold used to define the structures (cf. our discussion below). For low thresholds (40 G), the dimension increases from $D \approx 1.50$ at small areas to $D \approx 1.57$ at large areas. This area dependence gets larger with higher magnetic thresholds. The dimensions from the MDI data are definitely larger than the value $D=1.21$ found in the present study. We will see the origin of the differences, at least part of it below in Sect. 5.4.

The HR magnetograms in Meunier's (1999) work are closer in spatial resolution to the present analysis than her FD data. For the HR magnetograms, she finds again a dependence on the area and on the magnetic threshold, the dimension varying between $D \approx 1.40$ at small areas and $D \approx 1.65$ at large areas with a threshold of $40 \mathrm{G}$.

\subsubsection{Seeing dependence and noise}

From the speckle reconstruction procedure one obtains the Fried parameter $r_{0}$, which was in the range of $7 \mathrm{~cm}$ to $16 \mathrm{~cm}$. We report without showing that a dependence of the dimension on $r_{0}$ is not noticeable. When we omit fields with less than 20 magnetic structures above the threshold of 120 Gauss the scatter of the dimension is $\Delta D \approx 0.15$ (for details see Janßen 2003). The independence of $D$ on $r_{0}$ gives confidence in the image reconstructions. The method gives consistent shapes of magnetic structures once the seeing conditions are satisfactory.

To test the sensitivity of the results on the noise, a random magnetic field, distributed according to a Gaussian, was added to few magnetograms. The standard deviation of the Gaussian was set equal to the detection limit of 50 Gauss. The dimension increased insignificantly by $\Delta D=0.02$. We conclude that noise is not important in our results.

\subsection{Fractal dimension from numerical simulations}

The area covered by the numerical simulations is approximately six times smaller than the FOV of the observations. This is well compensated by the 3.5 times smaller pixel size than in the the observations. Furthermore, the spatial resolution of the observed magnetograms is (approximately) 0.' 4, which is a factor 14 larger than the corresponding pixel size of the simulations. Apart from the general question on the fractal structure of the simulated magnetograms, it is also of interest to compare magnetograms whose geometric scale differs by an order of magnitude (cf. Stenflo \& Holzreuter 2002, 2003a,b).

For a statistically significant sample we used 10 independent snapshots, i.e. separated well in time, from a simulation which had started with a constant vertical magnetic field of 50 Gauss. Once the simulated magnetoconvection was fully developed strong fields with strengths up to 2000 Gauss were generated, like in solar network or plage regions. From the 10 magnetograms, that is from the maps of the magnetic field components along the line-of-sight, 1153 structures (containing at least six pixels) with $|B| \geq 120$ Gauss were found. Figure 5 shows the perimeter-area relation. We point out that the area of the pixels in this figure is smaller by a factor of 12 (or $\Delta \log (A)=1.08)$ than that of the observations in Fig. 4. The dimension of the simulations in Fig. 5 is $D=1.38$.

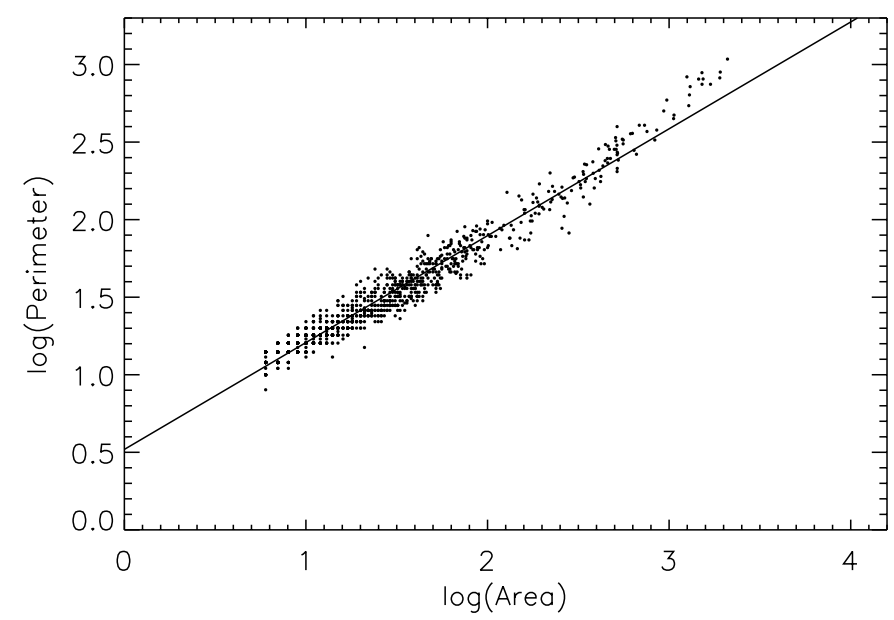

Fig. 5. Perimeter-area relation of magnetic structures obtained from numerical simulations with an initial vertical field of 50 Gauss. The fractal dimension $D=1.38 \pm 0.07$ is obtained. Note that the areas here are 12 times smaller than in Fig. 4 (since the pixel size was $20.8 \mathrm{~km}$, which is $\sqrt{12}$ times smaller than in Fig. 4).

We also see in Fig. 5 the tendency of an increased dimension for areas with $\log \left(A /\right.$ pixel $\left.^{2}\right) \geq 2.5$ as in the observations, cf. Fig. 4. But one should also remember that the pixel sizes in the two figures are very different. 


\subsection{Comparison of fractal dimensions from observations and from simulations}

\subsubsection{Spatial resolution}

The difference of fractal dimension $\Delta D=0.17$ between the simulated and the observed magnetograms is not at all negligible. It is much larger than the standard deviation of the observational error (cf. Fig. 3).

However, there exists an important difference between the determintation of the fractal dimension from the observations and from the simulations: The spatial resolution of the numerical simulations agrees with the pixel size, i.e. with the length of the yardstick $G$ (cf. Sect. 5.1). It does not agree for the observations. In the latter, the spatial resolution is approximately four times worse than the pixel size, or yardstick $G$. This mismatch decreases the fractal dimension. Assume that the small-scale structures were observed with low resolution of $5^{\prime \prime}$, say. The ensuing shapes will be much more roundish than those in Fig. 1 and the resulting fractal dimension will be close to $D=1.0$ when measured with $G=0$.' 1 . It is an important condition for the determination of $D$ that the length $G$ be not smaller than the spatial resolution. This has not been taken into account in the above measurements of $D$ from the observations, like in some earlier publications.

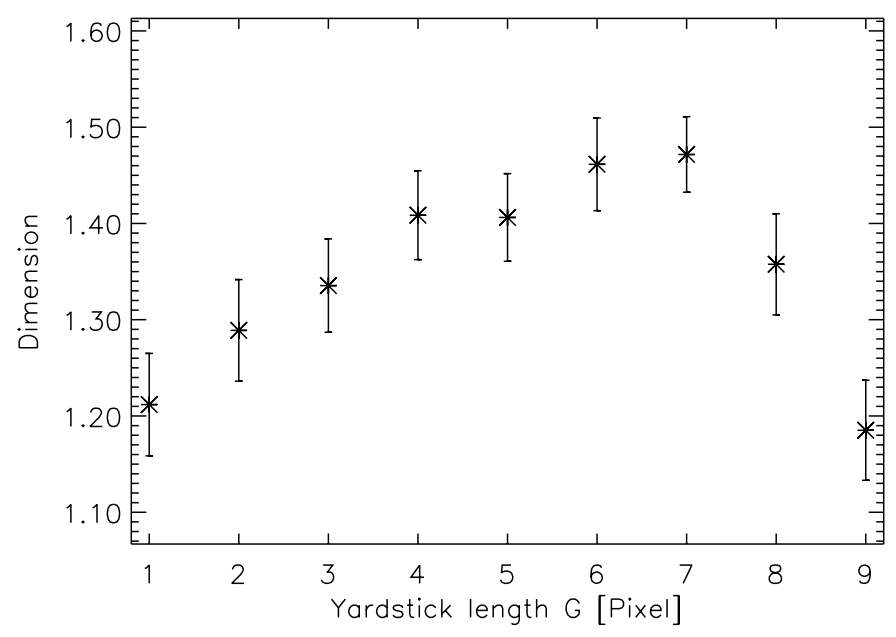

Fig. 6. Dependence of fractal dimension $D$ on the length of the yardstick $G$ for the observations, in units of the pixel size of $72 \mathrm{~km}$.

If we adapt $G$ to the spatial resolution the fractal dimension $D$ increases. This is demonstrated in Fig. 6 where the dependence of $D$ on $G$ is shown for the observations. When $G$ has reached the spatial resolution $(\approx 0,4)$ the dimension has attained $D=1.41$ in good agreement with the value from the simulations. $D$ remains essentially constant with increasing $G$ (cf. Sect. 5.1) and then decreases for $G \geq 8$ pixel because then the - relatively small - structures appear increasingly simpleshaped, more or less quadratic. Using the correct yardstick, adapted to the spatial resolution of our magnetograms, brings also the fractal dimension in agreement with the value found by Meunier (1999) from MDI HR magnetograms, at least for the small magnetic patches in her measurements.
As an additional test we may decrease the spatial resolution of the numerically simulated magnetograms by smearing them with a Gaussian with full width at half maximum of 4 pixel. The resulting fractal dimension for the simulation becomes then $D=1.27$ in closer agreement with the observations than the above value of 1.38 .

The agreement between observations and numerical simulations had not to be expected a priori. If not incidental it shows

1. that numerical simulations of magnetoconvection have reached a high degree of realism;

2. and that, although the scales of the simulations and the observations differ by an order of magnitude, the structures exhibit geometric similarity well below the resolution of telescopes existing today or to be constructed in the near future, as conjectured by Stenflo \& Holzreuter (2003b).

\subsubsection{Threshold of magnetic field strength}

Up to now, for the perimeter and area determination, we have set the lower limit of the line-of-sight component of the magnetic field $\left|B_{\text {eff }}\right|=120$ Gauss. Figure 7 depicts the variation of the fractal dimension $D$ when this lower limit is changed.

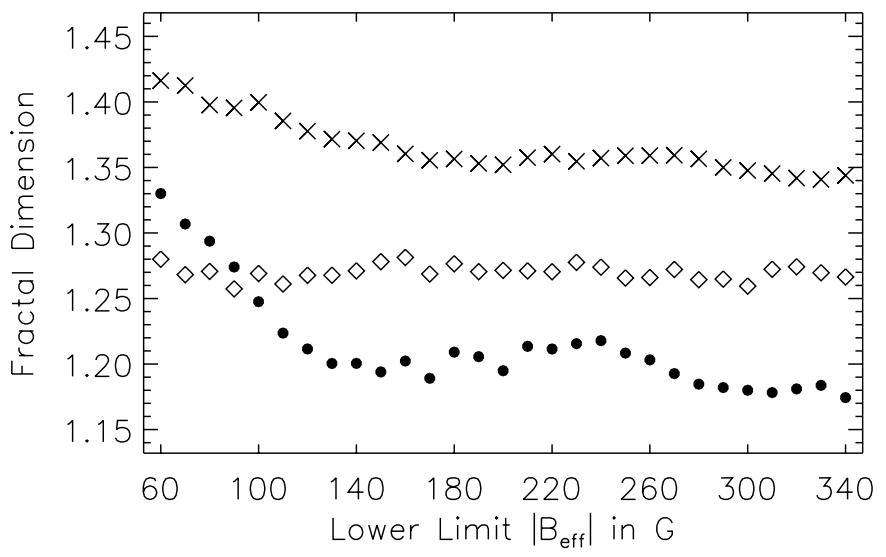

Fig. 7. Dependence of fractal dimension $D$ on the lower limit of $|B|$ for determining perimeter and area. $\bullet \bullet$ : observations; $\times \times \times$ : simulations, original resolution; $\diamond \diamond \diamond$ : simulation, after smearing with a Gaussian (cf. Sect. 5.4.1).

The strongest changes occur in the observational results at low limiting field strengths. There, $D$ decreases from 1.33 to 1.20 when the threshold rises from 60 Gauss to 120 Gauss. Meunier (1999) has found a similar trend for MDI FD magnetograms at small areas. But contrary to our results with only a weak dependence at large magnetic thresholds, the trend continues for large thresholds in Meunier's measurements and it reverses at large areas. For her HR data the dependence of the fractal dimension is much less pronounced. In our case, tests have shown that the high dimension values at low field strengths are likely a result of the noise in the magnetograms (see Sect. 5.2.2). For 120 Gauss and larger, the noise becomes negligible and the small decrease of $D$ for large thresholds is presumably due to more small-scale, more concentrated, and compact structuring of the flux regions than the shape of the 
weaker flux areas. The strong flux features thus possess either intrinsically simpler shapes or are more subject to seeing and attain smoother boundaries from the limited resolution. This view is supported by the observations of Hartje \& Kneer (2002) that, in enhanced network, the regions with strongest flux have the smallest extent.

Figure 7 shows that the simulations with their original resolution exhibit a similar trend of decreasing dimension for increasing field strength. This supports the view that strong fields possess intrinsically a compact structure. If we smear the simulated magnetograms with a Gaussian seeing function the dependence of $D$ on the limiting field strength almost disappears. This is expected when we assume that weak flux structures have a more complex structure than strong flux features. The complexity of the former is diminished by the "seeing".

\subsubsection{Break of similarity}

We had mentioned in the Introduction that many researchers have found a change of fractal dimension of granules occurring at approximately 1. ". 5 . For larger structures the dimension is large, $D \approx 2$. A change of dimension is also indicated in our magnetic field observations (cf. Fig. 4, Sect. 5.2.1). For $\log \left(A /\right.$ pixel $\left.^{2}\right) \geq 2.5$ we obtain from a sample of 42 structures a fractal dimension $D=1.47$. We estimate the structural length $l$ where the change occurs as

$l \approx\left(10^{2.5}\right)^{1 / 2}$ pixel $\approx 11^{\prime \prime} 8$,

i.e. in the same range as found for the granulation. If this finding is confirmed on the basis of higher statistical significance it shows a break of similarity at a certain structural length and hints to a change of the magnetoconvective process at this scale.

Instead of finding a break of fractal dimension at a certain scale, Meunier (1999) reports on a continuous increase of dimension with increasing area of the magnetic stuctures. This indicates possibly, instead of a change of the magnetoconvective process, an increased complexity with increased depth in the solar interior where the magnetic flux from large, compact flux ropes is separated. This would give an increased time for interaction with the convection, for diffusion and instabilities, like the flute instability, to work on the magnetic flux tubes.

Surprisingly, the simulations indicate a similar break as our measurements, again at $\log \left(A / \mathrm{pixel}^{2}\right) \approx 2.5$. Sixty-seven data points above this level give $D=1.90$. The according structural length is $l \approx 370 \mathrm{~km}$, very different from the length where the break occurs in the observations. At present, we cannot expand further on this point. But we assume, as a working hypothesis for further studying the break, a relation between this length and the lower boundary (at $z=-800 \mathrm{~km}$ ) of the simulation box.

\section{Conclusions}

Our starting point was the geometric similariy of complex magnetic structures on the Sun over a large range of scales. The property of magnetic self-similarity was studied by, e.g.,
Meunier (1999) and has recently been taken up again by Stenflo \& Holzreuter $(2002,2003 \mathrm{a}, \mathrm{b})$. The idea is that the complexity and scale invariance of magnetic structures is caused by magnetoconvection.

Thus, following this conception, we performed fractal analyses - by means of the perimeter-area relation - of observed magnetograms with high spatial resolution (0.'4-0.'5) and of numerical simulations of magnetoconvection. The agreement between the results from these two data sets is very good. Once the measuring scale, i.e. the yardstick, for the observations is adapted to their spatial resolution we obtain fractal dimensions $D=1.41 \pm 0.05$ for the observations and $D=1.38 \pm 0.07$ for the simulations.

The agreement is remarkable and astounding: The observations have a pixel length, after adaption for limited resolution, of approximately $300 \mathrm{~km}$ on the Sun, while the pixels in the numerical simulations are 15 (!) times smaller. Thus, the simulations represent the continuation of the observed magnetograms to very small scales not observable with today's solar telescopes. The finding leads to two conclusions:

1. The agreement between the fractal dimensions from the simulations and the observations shows that the simulations have reached a high degree of realism. This is not restricted to the convective phenomenon itself. It includes the complexity of the magnetic field structures seen at the solar surface.

2. The result supports the conjecture by Stenflo \& Holzreuter (2003b) that the complexity and geometric similarity of magnetic structures will continue when zooming into smaller and smaller scales.

Despite the expectation of self-similarity at still smaller scales we are certainly curious about the results to be gained from next generation solar telescopes. It is important to continue such studies with very high spatial resolution to prove or disprove the expectation. Which geometric structure of the solar magnetic field will be found once we achieve a spatial resolution of 0 '. 1 and smaller? Similarly, which structuring will arise from numerical simulations at scales still much smaller than the lower limit of $20 \mathrm{~km}\left(\cong 0{ }^{\prime} 028\right)$ used here?

In our study, the question about a break of similarity at a geometric scale of $l=1^{\prime \prime} 5 \ldots 2^{\prime \prime} \cdot 0$ arose. It is indicated by a change of fractal dimension at these scales. Such a change occurs also in the simulated data, but at a much smaller scale of $l \approx 0$ '.5. We have no established explanation and must defer the question to further investigations. The finding by Meunier (1999) of a continuous increase of the fractal dimension, instead of a change at a certain scale, with increasing magnetic structural size is worth mentioning again. It indicates that geometric (self-)similarity does not strictly hold, at least for the large structures studied by Meunier (1999). The change of dimension may be related to the depth of the origin of the magnetic structures in the solar interior.

The fractal dimension $D \approx 1.40$ found here for magnetic structures is, beyond the statistical error, larger than that from the perimeter-area relation for granular intensity structures with $D \leq 1.30$. There may be two reasons for it: 1) the magnetic surface structures, after having undergone magnetoconvective 
processes in deep subphotospheric layers, are indeed more complex than the granular intensity pattern at the surface. 2) Possibly, the yardstick for the measurement of the granular perimeters was not adapted to the spatial resolution. This latter view advocates for a re-analysis of the granular fractal perimeters.

Finally, there is no doubt on the complex, close to selfsimilar structuring of magnetic fields on the Sun. The agreement between the fractal dimensions from observations and simulations found here gives confidence that all necessary processes are included in the simulations. This gives good promises that we can learn from future simulations of magnetic field dynamics about the magnetic structuring of the solar atmosphere and of stellar atmospheres in general. Realistic models are needed to understand the magnetically coupled system from the solar/stellar interior through the atmosphere out to the corona, solar wind, and heliosphere.

Acknowledgements. We thank E. Landi Degl'Innocenti and M. Schüssler for helpful discussions and comments on the manuscript. KJ thanks the Deutsche Forschungsgemeinschaft (DFG) for a one year fellowship of the Graduiertenkolleg GRK 140 Strömungsinstabilitäten und Turbulenz. Financial support by DFG through grants KN 152/251 and SCHU 500/7-3 is gratefully acknowledged. The Vacuum Tower Telescope is operated by the Kiepenheuer-Institut für Sonnenphysik, Freiburg, at the Spanish Observatorio del Teide of the Instituto de Astrofísica de Canarias.

\section{References}

Balke, A. C., Schrijver, C. J., Tarbell, T. D., \& Zwaan, C. 1993, Sol. Phys., 143, 215

Bendlin, C. 1993, Ph.D. Thesis, Göttingen University

Bendlin, C., \& Volkmer, R. 1995, A\&AS, 112, 371

Bendlin, C., Volkmer, R., \& Kneer, F. 1992, A\&A, 257, 917

Bovelet, B., \& Wiehr, E. 2001, Sol. Phys., 201, 13

de Boer, C. R. 1996, A\&AS, 120, 195

de Boer, C. R., Kneer, F., \& Nesis, A. 1992, A\&A, 257, L4

Domínguez Cerdẽna, I., Kneer, F., \& Sánchez Almeida, J. 2003, ApJ, 582, L55

Domínguez Cerdẽna, I., Sánchez Almeida, \& Kneer, F. 2003, A\&A, 407,741
Greimel, R., Brandt, P. N., Guenther, E., \& Mattig, W. 1990, Vistas Astron., 33, 413

Hartje, B., \& Kneer, F. 2002, A\&A, 385, 264

Hirzberger, J., Vázquez, M., Bonet, J. A., Hanslmeier, A., \& Sobotka, M. 1997, ApJ, 480, 406

Janßen, K. 2003, Ph.D. Thesis, Göttingen University

Karpinsky, V. N. 1990, in Solar Photosphere: Structure, Convection, and Magnetic Fields, ed. J. O. Stenflo (Dordrecht, Holland: Kluwer), IAU Symp., 138, 67

Keller, C. U., von der Lühe, O. 1992, A\&A, 261, 321

Koschinsky, M., Kneer, F., \& Hirzberger, J. 2001, A\&A, 365, 588

Krieg, J., Wunnenberg, M., Kneer, F., Koschinsky, M., \& Ritter, C. 1999, A\&A, 343, 983

Mandelbrot, B. 1977, Fractals (San Francisco: Freeman)

Meunier, N. 1999, ApJ, 515, 801

Rees, D. E., \& Semel, M. D. 1979, A\&A, 74, 1

Roudier, T., \& Muller, R. 1987, Sol. Phys., 107, 11

Schüssler, M. 2003, in Third International Workshop on Solar Polarization, ed. J. Trujillo Bueno, \& J. Sánchez Almeida, ASP Conf. Ser., in press

Schrijver, C. J., Zwaan, C., Balke, A. C., Tarbell, T. D., \& Lawrence, J. K. 1992, A\&A, 253, L1

Semel, M. D. 1967, Ann. Astrophys., 30, 513

Stenflo, J. O., \& Holzreuter, R. 2002, in Magnetic Coupling of the Solar Atmosphere, ed. H. Sawaya-Lacoste, ESA Publ. SP-505, 101

Stenflo, J. O., \& Holzreuter, R. 2003a, in Current Theoretical Models and Future High Resolution Solar Observations: Preparing for ATST, ed. A. A. Pevtsov, \& H. Uitenbroek, ASP Conf. Ser., 286, 169

Stenflo, J. O., \& Holzreuter, R. 2003b, Astron. Nachr., 324, 397

Tarbell, T. D., Ferguson, S., Frank, Z., et al. 1990, in Solar Photosphere: Structure, Convection, and Magnetic Fileds, ed. J. O. Stenflo (Dordrecht, Holland: Kluwer), IAU Symp., 138, 147

Vögler, A. 2003, PhD thesis, Göttingen University

Vögler, A., \& Schüssler, M. 2003, Astron. Nachr., 324 (4), 399

Vögler, A., Shelyag, S., Schüssler, M., et al. 2003, in Modelling of Stellar Atmospheres, ed. N. E. Piskunov, W. W. Weiss., \& D. F. Gray, IAU Symp. 210, ASP Conf. Ser., in press von der Lühe, O. 1984, J. Opt. Soc. Am., A1, 510

Weigelt, G. P. 1977, Opt. Comm., 21, 55

Weigelt, G. P., \& Wirnitzer, B. 1983, Opt. Lett., 8, 389 